eclampsia (12\%). The times when fields 43 (ie, death during pregnancy, labour or miscarriage) and 44 (during the puerperium) of the death certificate showed potential reasons for sub-registration, beyond not contributing to the selection of the deaths investigated. Conclusion The analysis of the deaths investigated enabled us to clarify all the cases of maternal morbi-mortality. This study showed how joint surveillance action together with that of the Committee enhances the quality of the information and contributes to the advance of maternal health.

\section{P1-290 ESTIMATES OF AVOIDABLE DEATHS BY FAECAL OCCULT BLOOD TEST (FOBT) SCREENING FOR COLORECTAL CANCER IN THE EU}

doi:10.1136/jech.2011.142976e.82

\begin{abstract}
${ }^{1} \mathrm{P}$ Pisani, ${ }^{*} \mathrm{C}$ Herrmann, ${ }^{2} \mathrm{D}$ Sighoko, ${ }^{2} \mathrm{~T}$ Lignini, ${ }^{2} \mathrm{~S}$ Ducarroz, ${ }^{2} \mathrm{~L}$ von Karsa. ${ }^{1}$ Childhood Cancer Registry of Piedmont, Cancer Epidemiology Unit University of Torino, CPO Piemonte, Turin, Italy; ${ }^{2}$ International Agency for Research on Cancer, Lyon, France
\end{abstract}

Introduction RCTs have demonstrated CRC screening efficacy. However, programme implementation requires substantial resources. Reliable estimates of the potential screening impact in a population would facilitate timely decisions about establishing programmes. Many countries lack the capacity and detailed knowledge of the distribution of the disease in the population for complex modelling, particularly medium-resource countries. Simple methods for estimating the future impact of CRC screening in such settings would be a useful tool in cancer control planning.

Methods For the $27 \mathrm{EU}$ countries, population projections by country, sex and quinquennium were obtained from the UNpopin database. Country-, sex- and age-specific mortality rates were obtained from GLOBOCAN2002. The method requires stating parameters for the following factors: screening interval, age at screening attendance, participation rate and programme duration. Estimates for these parameters were derived from FOBt RCT results; simultaneous programme introduction throughout a country and high quality management were assumed.

Results 600000 to 1.05 million CRC deaths could be avoided over 25 years in the EU depending on the screening interval and compliance rate, for programmes offered to the 50-74-year-old population.

Conclusions A method for estimating the population impact of CRC screening has been developed which requires minimum epidemiologic and technical support. The accuracy of the method should be assessed by comparing these preliminary results with sophisticated modelling approaches and with up-to-date estimates of CRC burden in populations in which screening coverage is known. Countries contemplating CRC screening, but lacking detailed knowledge of the disease burden, should develop this capacity in the early translational phase of programme planning.

\section{P1-291 INTEGRATED DISEASE SURVEILLANCE AND RESPONSE: PERFORMANCE ASSESSMENT IN A TERTIARY NIGERIAN HOSPITAL}

doi:10.1136/jech.2011.142976e.83

0 Popoola, ${ }^{*}$ A Fatiregun, S Ige. University College Hospital, Ibadan, Oyo state, Nigeria

Introduction Efficient case reporting via the Integrated Disease Surveillance (IDS) system is one of the main strategies to control of Lassa fever. Diagnosing Lassa fever is complicated by similarity of case presentation with common environmental causes of fever like malaria and typhoid, and limited laboratory capacity for identification.
Objectives This study was conducted to assess trainee doctors' capacity to recognise and IDS report Lassa as well as institutional mechanisms for IDS.

Methods Mixed methods, questionnaire survey of 260 doctors and key informant interviews with record officials.

Results Over $65 \%$ of respondents correctly identified case definition of Lassa fever and most evidenced positive attitudes towards requirement of reporting despite work and time constraints. Relevant knowledge for IDS reporting was however poor. Over 30\% of respondents did not know they were obliged to report these cases to public health authorities. About $50 \%$ did not realise a single suspected case constituted an emergency worth reporting and only $12 \%$ knew which forms to use. None of the physicians who had patients with symptoms matching case definition reported these to the local PHC office. Prior knowledge of the IDS system was due mainly to undergraduate learning experience. The institutional reporting mechanism had lapses IDS manuals were not available in any of the clinical practice areas, reporting forms and posters of case definitions were absent and clinicians did not maintain registers of patient diagnosis.

Conclusion Lassa fever is unlikely to be reported through the IDS system. Efforts should target improving institutional compliance with regulations and increased education targeting physicians.

\section{P1-292 ASSOCIATION BETWEEN STRESS, PERSONALITY TRAITS AND SLEEP BRUXISM IN CHILDREN: A POPULATION-BASED CASE-CONTROL STUDY}

doi:10.1136/jech.2011.142976e.84

${ }^{1} \mathrm{~J} M$ Serra-Negra, ${ }^{1} \mathrm{~S} M$ Paiva, ${ }^{2} \mathrm{M}$ L Ramos-Jorge, ${ }^{1} \mathrm{C}$ E Flores-Mendoza, ${ }^{1}$ I A Pordeus.* ${ }^{1}$ Universidade Federal de Minas Gerais, Belo Horizonte, Minas Gerais, Brazil; ${ }^{2}$ Universidade Federal dos Vales do Jequitinhonha e Mucuri, Diamantina, Minas Gerais, Brazil

Introduction Sleep bruxism is an unusual orofacial disorder affecting both children and adults. Its consequences include temporomandibular disorder, muscle pain, periodontal problems, tooth wear and tooth loss. Its aetiology remains unclear, and a multifactorial nature has been attributed to pathophysiological, psychological and morphologic aspects. Most studies carried out so far involve adults and few have investigated younger groups. Hence this study aimed to assess the association between stress levels, personality traits and sleep bruxism in children.

Methods A population-based case-control study (proportion of 1:2) was carried out involving 120 children with sleep bruxism and 240 children without this disorder aged between 7 and 11 years. The sample was randomly selected from schools in the city of Belo Horizonte, Brazil. The following instruments were employed for the data collection: questionnaire administered to parents; Child Stress Scale (CSS); and Neuroticism and Responsibility scales of the Big Five Questionnaire for Children (BFQ-C). Psychological tests were administered and evaluated by psychologists. Sleep bruxism was diagnosed from parents' reports. The $\chi^{2}$ test, binary and multivariate logistic regression were applied for the statistical analysis.

Results In the adjusted logistic model, children with a high level of stress due to psychological reactions $(\mathrm{OR}=1.8 ; 95 \% \mathrm{CI} 1.1$ to 2.9$)$ and high sense of responsibility ( $O R=1.6 ; 95 \%$ CI 1.0 to 2.5 ) had a nearly twofold greater chance of having sleep bruxism in comparison to those with low levels of these psychological traits.

Conclusion High levels of stress and responsibility are associated factors for the development of sleep bruxism among children.

Funding FAPEMING, CNPq 